\title{
ON A CLASS OF TRANSFORMATIONS WHICH HAVE UNIQUE ABSOLUTELY CONTINUOUS INVARIANT MEASURES \\ BY \\ ABRAHAM BOYARSKY ${ }^{1}$ AND MANNY SCAROWSKY
}

\begin{abstract}
A class of piecewise $C^{2}$ transformations from an interval into itself with slopes greater than 1 in absolute value, and having the property that it takes partition points into partition points is shown to have unique absolutely continuous invariant measures. For this class of functions, a central limit theorem holds for all real measurable functions. For the subclass of piecewise linear transformations having a fixed point, it is shown that the unique absolutely continuous invariant measures are piecewise constant.
\end{abstract}

1. Introduction. In [1], Lasota and Yorke established the existence of absolutely continuous invariant measures for the class of piecewise $C^{2}$ point transformations (see Definition 1 below) $\tau$ from an interval into itself and satisfying $\inf _{x}|d \tau / d x|>1$, wherever the slope exists. In [2], it was shown that if a function in this class has $n$ discontinuities, then there are at most $n$ independent, absolutely continuous invariant measures under $\tau$.

The purpose of this paper is to show that a certain subclass $\mathcal{C}$ of the aforementioned class of functions possesses unique absolutely continuous invariant measures. Functions in $\mathcal{C}$ are characterized by a communication property on the intervals that form the partition of the function. Using the method of symbolic dynamics [3], this property establishes the existence of a dense orbit, from which uniqueness follows.

In [9], it is shown that a central theorem holds for the $C^{2}$ transformations of [1] for a class of real Hölder functions. In $\$ 5$, we obtain a central limit theorem for transformations in $\mathcal{C}$ in a simpler way, and the theorem holds for all real measurable functions. In $\$ 6$, it is shown that if $\tau \in \mathcal{C}$ is piecewise linear and has a fixed point, then the unique absolutely continuous measure invariant under $\tau$ is piecewise constant.

2. Uniqueness theorem. Denote by $\left(\varrho_{1},\|\|\right)$ the space of all integrable functions on the interval $J=[a, b]$. Let $m$ denote the Lebesgue measure on $J$. Let $\tau: J \rightarrow J$ be a measurable nonsingular transformation. By "nonsingular"

Received by the editors April 10, 1978 and, in revised form, August 1, 1978. AMS (MOS) subject classifications (1970). Primary 28A65; Secondary 60F05.

'The research of this author was supported by a National Research Grant. No. A-9072. 
we mean that $m\left(\tau^{-1}(A)\right)=0$ whenever $m(A)=0$ for $A$ a measurable set. A measure $\mu$ is said to be invariant under $\tau$ if for all measurable sets $A \subset J$, we have $\mu(A)=\mu\left(\tau^{-1}(A)\right)$, where $\tau^{-1}(A)=\{x \in J: \tau(x) \in A\}$. $\mu$ is absolutely continuous if there exists an $f \in \mathfrak{L}_{1}, f(x) \geqslant 0$, such that $\mu(A)=\int_{A} f(x) d x$ for every Lebesgue measurable set $A \subset J$. We refer to $f$ as the invariant density (of $\mu$ ) under $\tau$. It is well known [1] that the invariant density $f$ is a fixed point of the Frobenius-Perron operator $P_{\tau}: \mathscr{L}_{1} \rightarrow \mathscr{L}_{1}$ defined by

$$
P_{\tau} f(x)=\frac{d}{d x} \int_{\tau^{-1}([0, x])} f(s) d s .
$$

Definition 1. A transformation $\tau: J \rightarrow J$ is called piecewise $C^{r}$ if there exists a partition $G=\left\{\left(a_{0}, a_{1}\right),\left(a_{1}, a_{2}\right), \ldots,\left(a_{N-1}, a_{N}\right)\right\}, a_{0}=a, a_{N}=b$, such that for each $i \in\{1, \ldots, N\}, \tau$ restricted to $\left(a_{i-1}, a_{i}\right)$ is a $C^{r}$ function which can be extended to the closed interval $\left[a_{i-1}, a_{i}\right]$ as a $C^{r}$ function. $\tau$ need not be continuous at the points $a_{i}$.

Definition 2. Let $Q$ be the partition points of the partition 9 . We say the transformation $\tau: J \rightarrow J$ takes partition points into partition points if $\tau(Q) \subset$ $Q$. If $\tau$ is discontinuous, we require $\tau\left(a_{i}^{-}\right)$and $\tau\left(a_{i}^{+}\right)$to be in $Q$.

The transformation $\tau$ is often called a Markov map [14].

Definition 3. We say that the partition 9 has the communication property under the transformation $\tau: J \rightarrow J$ if for any $I_{i}, I_{j} \in \mathcal{G}$, there exist integers $n$ and $m$ such that $I_{i} \subset \tau^{n}\left(I_{j}\right)$ and $I_{j} \subset \tau^{m}\left(I_{i}\right)$.

A point transformation $\tau: J \rightarrow J$ is in class $C$ if it satisfies the following conditions for the fixed partition 9 :

(1) $\tau$ is piecewise $C^{2}$ with respect to 9 ,

(2) $\inf _{x \in J}|d \tau / d x|>0$ and $\inf _{x \in J}\left|d \tau^{l} / d x\right|>1$ for some integer $l$,

(3) $\tau^{l}$ takes partition points into partition points,

(4) the partition $G$ has the communication property under $\tau$.

In view of [5], condition (1) can be replaced by $\left(1^{\prime}\right): \tau$ is piecewise $C^{1}$ and $\tau_{i}=\left.\tau\right|_{\left(a_{i-1}, a_{i}\right)}$ has the property that $1 /\left|\tau_{i}^{\prime}\right|$ is of bounded variation on $\left[a_{i-1}, a_{i}\right]$.

Without loss of generality, we shall assume that $\tau(Q) \subset Q$. If this were not the case, we would define a new partition $\bar{g}$ whose partition points $\bar{Q}$ would satisfy $\tau(\bar{Q}) \subset \bar{Q}$. It is clear that properties (1) and (2) would remain valid for the new partition. Note that condition (3) is equivalent to the statement that the partition points are eventually periodic. The point $x \in J$ is an eventually periodic point of the function $\tau$ if there exists an $n=n(x)$ such that $\tau^{n}(x)$ is periodic where $\tau^{n}=\tau \circ \tau \circ \cdots \circ \tau, n$ times.

Our first objective is to show that a large class of transformations $\tau$ admit a dense orbit. To do this, we use symbolic dynamics [3]. We associate with each of the $N$ intervals $\left[a_{i-1}, a_{i}\right]$ a symbol such as $\alpha, \beta, \gamma, \ldots$ and code the orbit by a sequence $\langle x\rangle=. \alpha \beta \gamma \cdots$, if $x \in I(\alpha), \tau(x) \in I(\beta), \tau^{2}(x) \in I(\gamma), \ldots$ where $I(\alpha)$ is the interval in $\mathscr{G}$ whose symbol is $\alpha$. Note that this coding is well 
defined except for possibly the points $\tilde{Q}$ eventually entering the partition points $Q$. We shall need the following three lemmas.

LEMMA 1. Let $\tau: J \rightarrow J$ be piecewise monotonic and satisfy condition (2) defining class $\mathcal{C}$. Then $\langle x\rangle=\langle y\rangle$ implies $x=y$.

Proof. Assume $x \neq y$, but $\langle x\rangle=\langle y\rangle$. By condition (2), there exists an integer $l$ such that

$$
\left|\frac{d \tau^{l}}{d x}\right| \geqslant d>1
$$

Now, $\langle x\rangle=\langle y\rangle$ implies that $\tau^{n l+i}(x)$ and $\tau^{n l+i}(y)$ belong to the same interval for each $n$ and $i, 0 \leqslant i \leqslant l-1$, but (1) implies that

$$
\left|\tau^{n l+i}(x)-\tau^{n l+i}(y)\right| \geqslant d^{n} c^{i}|x-y| \rightarrow \infty, \quad i \leqslant n-1,
$$

as $n \rightarrow \infty$, where $c=\inf \left|\tau^{\prime}\right|$. This is a contradiction. Q.E.D.

The converse of Lemma 1 is true if $x, y \notin \tilde{Q}$. With a convention for coding points $x \in \tilde{Q}$, the converse of Lemma 1 is true also for $x, y \in \tilde{Q}$.

LEMMA 2. Let $\tau$ be as in Lemma 1. If $\sigma=\alpha_{1} \alpha_{2} \alpha_{3} \cdots$ is a sequence with the property that $\tau\left(I\left(\alpha_{k}\right)\right) \supset I\left(\alpha_{k+1}\right), k=1,2,3, \ldots$, then there exists $a$ unique $x \in J$ such that $\langle x\rangle=\sigma$.

Proof. Let $J_{i}=\left\{x \in J: x \in I\left(\alpha_{1}\right), \ldots, \tau^{i-1}(x) \in I\left(\alpha_{i}\right)\right\}$. Each $J_{i}$ is a nonempty closed interval since $\tau$ is monotonic on each interval, and $J_{i} \supset J_{i+1}$ by the hypothesis. Thus $\bigcap_{i>0} J_{i} \neq \varnothing$, and by Lemma $1, \cap_{i>0} J_{i}$ consists of a single point. Q.E.D.

LEMMA 3. Let $\tau$ be as in Lemma 1. Let $\xi \subset 9$ be a collection of intervals satisfying the communication property: for $I_{1}, I_{2} \in \xi$ there exist $n$ and $m$ such that $I_{1} \subset \tau^{m}\left(I_{2}\right)$ and $I_{2} \subset \tau^{n}\left(I_{1}\right)$. Assume $\xi$ contains at least two intervals and let $V=\cup_{I \in \xi} I$. Then there exists an $x \in V$ such that $\left\{\tau^{i}(x)\right\}$ is dense in $V$.

(Note that if $\tau$ satisfies condition (4), there exists a dense orbit in all of $J$.)

Proof. Consider the set of all possible finite sequences $\alpha_{1} \alpha_{2} \cdots \alpha_{k}$, where $I\left(\alpha_{j}\right) \in \xi, j=1,2, \ldots, k$, and $\tau\left(I\left(\alpha_{j}\right)\right) \supset I\left(\alpha_{j+1}\right), \quad 1<j \leqslant k-1, k=$ $1,2,3, \ldots$ This set is countable. Let $S_{1}, S_{2}, S_{3}, \ldots$ be an enumeration, and form the sequence

$$
\langle x\rangle=\cdot S_{1} T_{1} S_{2} T_{2} S_{3} T_{3} \cdots,
$$

where the $T_{i}$ are finite sequences joining the last symbol of $S_{i}$ to the first symbol of $S_{i+1}$. That this can be done follows from the assumption that there exists $n$ such that $\tau^{n}(I(\alpha)) \supset I(\beta)$ for $I(\alpha), I(\beta) \in \xi$. Thus, by Lemma 2, a real $x$ exists corresponding to the coding $\langle x\rangle$.

Now, given $y \in V$ and $\varepsilon>0$, we claim there exists $n$ such that $\left|\tau^{n l}(x)-y\right|$ $<\varepsilon$. To see this, note that for any $m$, the symbol $S$ corresponding to 
$y \in I(\alpha), \tau(y) \in I(\beta), \ldots, \tau^{m}(y) \in I(\gamma)$ occurs in the coding of $x$. This implies that for some $n, \tau^{n l+i}(x)$ and $\tau^{i}(y)$ belong to the same intervals, $i=0,1, \ldots, m l$. Now

$$
\begin{aligned}
\left|\tau^{n l}(x)-y\right| & \leqslant \frac{1}{d}\left|\tau^{(n+1) l}(x)-\tau^{l}(y)\right| \\
& \leqslant \frac{1}{d^{m}}\left|\tau^{(n+m) l}(x)-\tau^{m l}(y)\right| \leqslant \frac{2 M}{d^{m}}<\varepsilon
\end{aligned}
$$

for $m$ sufficiently large, where $M=\max _{x \in J} \tau(x)$. Thus the orbit of $x$ is dense in $V$.

REMARK. There are uncountably many ways of ordering $S_{1}, S_{2}, S_{3}, \ldots$ and, in general, these result in uncountably many distinct sequences. Thus there are, in general, uncountably many such $x$.

THEOREM 1. Let $\tau \in C$. Then $\tau$ has a unique absolutely continuous invariant measure.

Proof. From [1], we know there exists an absolutely continuous measure invariant under $\tau$. Let us assume there are two such measures with densities $f_{1}$ and $f_{2}$. In view of [2, Lemma 2.6], the following two facts follow: (1) there exist two invariant densities $f_{1}^{*} \geqslant 0, f_{2}^{*} \geqslant 0,\left\|f_{1}^{*}\right\|=1,\left\|f_{2}^{*}\right\|=1$, such that $S_{1}=\operatorname{spt} f_{1}^{*}$ and $S_{2}=\operatorname{spt} f_{2}^{*}$ are disjoint, where spt $f$ denotes the support of $f$, the set on which $f(x)$ is nonzero, and (2) $S_{i}, i=1,2$, is a union of disjoint closed intervals.

Now, let $x \in J$ be a point which has a dense orbit in $J$. By Lemma 3 such a point exists. Let $y_{i} \in$ int $S_{i}, i=1,2$, where int denotes interior. The denseness of the orbit $\left\{\tau^{n}(x)\right\}$ implies there exist points $z_{1}=\tau^{n_{1}}(x)$ and $z_{2}=\tau^{n_{2}}\left(z_{1}\right)$ such that $z_{1} \in$ int $S_{1}$ and $z_{2} \in$ int $S_{2}$. By the piecewise continuity of $\tau$ there exists an open ball $O_{1}$ centered at $z_{1}$ and in $S_{1}$ such that for $z_{1} \in O_{1}$, $z_{2}=\tau^{n_{2}}\left(z_{1}\right) \in$ int $S_{2}$. But this contradicts the fact that $S_{1}$ and $S_{2}$ are invariant sets [2, Lemma 2.5], i.e. $\tau\left(S_{i}\right)=S_{i}$ a.e. Hence there exists only one absolutely continuous invariant measure under $\tau$. Q.E.D.

EXAMPLE 1. Consider the piecewise linear continuous function $\tau:[1,5] \rightarrow$ $[1,5]$ defined by $\tau(1)=3, \tau(2)=5, \tau(3)=4, \tau(4)=2, \tau(5)=1$, where $\tau$ is linear on each segment $[n, n+1], n=1,2,3,4$. The line segments have slope $-1, \pm 2$. The third iterate of $\tau, \tau^{3}$, however, has slopes $>1$ in absolute value for all segments. Let $Q=\{1,2,3,4,5\}$. Then it is clear that $\tau(Q) \subset Q$. Finally, it is easy to show that the partition $G=\{(1,2),(2,3),(3,4),(4,5)\}$ has the communication property under $\tau$. In fact, four is the maximum value of $n, m$ needed to ensure that $I_{i} \subset \tau^{m}\left(I_{j}\right)$ and $I_{j} \subset \tau^{n}\left(I_{i}\right)$, where $I_{i}, I_{j} \in \mathcal{G}$. Therefore $\tau \in \mathcal{C}$, and Theorem 1 applies, ensuring the uniqueness of the absolutely continuous measure under $\tau$. Theorem 1 of [2] claims that there are no more than three (linearly independent) invariant densities under $\tau$. Using 
the results of $\$ 6$ it is easy to show that the unique invariant density is given by

$$
f(x)=\left\{\begin{array}{lll}
2 / 7 & \text { on } & (1,2) \\
1 / 7 & \text { on } & (2,3) \\
2 / 7 & \text { on } & (3,4) \\
2 / 7 & \text { on } & (4,5)
\end{array}\right.
$$

To show that this is, indeed, an invariant density, one need only verify that $P_{\tau} f=f$.

EXAMPLe 2. Consider the nonlinear, piecewise monotonic function $\tau:[0,1]$ $\rightarrow[0,1]$ shown in Figure 1 , with $|d \tau / d x|>1$ where it is defined. It is easy to check that $\tau$ takes the partition points $\{0, .2, .4, .6, .8,1.0\}$ of the partition $\{(0, .2),(0.2,0.4),(0.4,0.6),(0.6,0.8),(0.8,1.0)\}$ into the same set. Also, the communication property follows readily from

$$
\tau\left(I_{1}\right)=\bigcup_{i=1}^{4} I_{i}, \quad \tau\left(I_{2}\right)=I_{3} \cup I_{4}, \quad \tau\left(I_{3}\right)=I_{3} \cup I_{4}, \quad \tau\left(I_{5}\right)=\bigcup_{i=1}^{5} I_{i} \text {. }
$$

Thus, by Theorem 1, there exists a unique absolute continuous invariant measure under $\tau$.

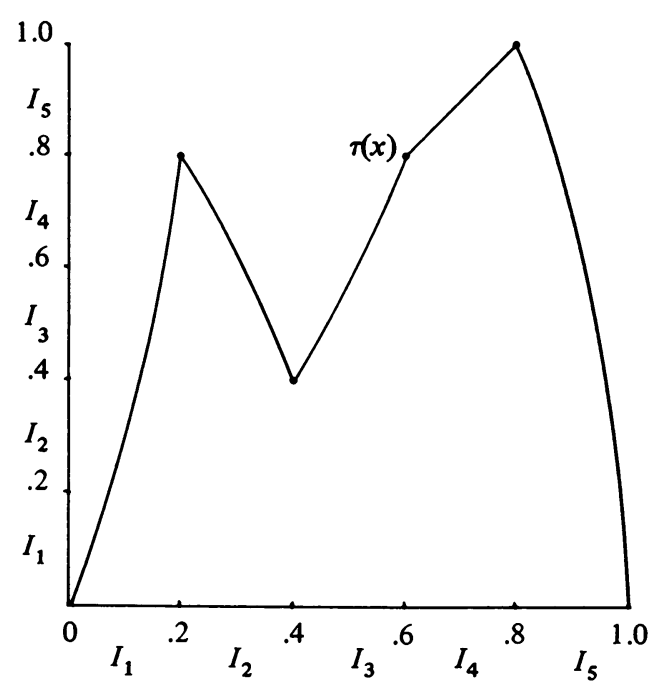

Figure 1

A nonlinear piecewise monotonic function with unique absolutely continuous invariant measure 
Let us define the discontinuous, nonlinear piecewise monotonic function $\tau_{1}$ as follows:

$$
\tau_{1}(x)=\left\{\begin{array}{l}
\tau(x), \text { as in Figure } 1, \quad x \in(0, .8), \\
\text { a smooth, nonlinear arc } \\
\text { with slope }>1 \text { in absolute } \\
\text { value joining }(.8, .8) \text { and } \\
(1.0,0),
\end{array} \quad x \in(.8,1.0)\right.
$$

Clearly $\tau_{1}\left(I_{5}\right)=\cup_{i=1}^{4} I_{i}$, and Theorem 1 applies once again to guarantee the existence of a unique absolutely continuous invariant measure under $\tau_{1}$.

3. Consequences of uniqueness. (I) The Birkhoff ergodic theorem applies. For almost all $x \in J$,

$$
\lim _{n \rightarrow \infty} \frac{1}{n} \sum_{i=0}^{n-1} g\left(\tau^{i}(x)\right)=\int_{J} g(x) f(x) d x,
$$

where $f$ is the unique density invariant under $\tau$ and $g$ is any bounded measurable function. (We note that if $f(x)>0$ on $J_{1} \subset J$, then for almost all $x,\left\{\tau^{n}(x)\right\}$ is dense in $J_{1}$.)

(II) Theorem 1 of [6] can be applied to approximate the unique density $f$ as closely as desired.

(III) Uniqueness is preserved for topologically conjugate transformations: We prove this as follows.

Let $\tau$ be a piecewise monotonic transformation and $h: J \rightarrow J$ a homeomorphism. Then $\tau_{1}=h \circ \tau \circ h^{-1}$ is a transformation from $J$ into $J$ and $\tau$ and $\tau_{1}$ are said to be topologically conjugate [3].

Proposition 1. Let $\tau \in \mathcal{C}$. Assume that for the homomorphism $h: J \rightarrow$ $J, h^{-1}$ is differentiable. Then $\tau_{1}=h \circ \tau \circ h^{-1}$ has a unique invariant density, and it is given by $f\left(h^{-1}(x)\right)\left(d h^{-1} / d x\right)$, where $f(x)$ is the unique invariant density under $\tau$ satisfying $f \geqslant 0,\|f\|=1$.

Proof. From Theorem 1, we know there exists a unique invariant density $f$ under $\tau$. Thus

$$
\int_{\tau^{-1}(A)} f d m=\int_{A} f d m
$$

where $m$ denotes the Lebesgue measure on $J$ and $A \subset J$ is a measurable set. Let $A=h^{-1}[a, x], x \in J$. Since $\tau_{1}=h \circ \tau \circ h^{-1}$, we have

$$
\int_{h^{-1} \tau_{1}^{-1}[a, x]} f d m=\int_{h^{-1}[a, x]} f d m .
$$

Without loss of generality, we assume that $h^{-1}$ is strictly increasing. Then the 
right-hand side of (1) can be expressed as

$$
\int_{a}^{h^{-1}(x)} f d m=\int_{a}^{x} f\left(h^{-1}(u)\right) \frac{d h^{-1}(u)}{d u} d m .
$$

Now, for all intervals $B=[a, x]$, it follows from (3) that

$$
\int_{h^{-1}(B)} f d m=\int_{B} f\left(h^{-1}(x)\right) \frac{d h^{-1}(x)}{d x} d m .
$$

Equation (4) is also valid for open sets $B$ and, from that, for all measurable sets $B$. Hence

$$
\int_{\tau_{1}^{-1}[a, x]} \bar{f} d m=\int_{[a, x]} \bar{f} d m,
$$

where $\bar{f}=\left(f \circ h^{-1}\right)\left(d h^{-1} / d x\right)$ is an invariant density under $\tau_{1}$. It remains to show that $\bar{f}$ is unique.

Assume it is not. Then there exist two densities $\bar{f}_{1}$ and $\bar{f}_{2}$, both invariant under $\tau_{1}$. By the foregoing argument, $\left(\bar{f}_{1} \circ h\right)(d h / d x)$ and $\left(\bar{f}_{2} \circ h\right)(d h / d x)$ are densities invariant under $\tau$. Requiring these densities to have $\mathfrak{L}_{1}$ norm equal to 1 and invoking the uniqueness of invariant densities under $\tau$ implies that $\bar{f}_{1}(h(u))=\bar{f}_{2}(h(u))$ for all $u$. Hence, $\bar{f}_{1}=\bar{f}_{2}$.

Actually, this proof shows that uniqueness of invariant densities is preserved under transformations of the form $h_{1} \circ \tau \circ h_{2}$, where $h_{1}$ and $h_{2}$ are homeomorphisms satisfying the conditions of Proposition 2.

4. Some matrix theoretic results. Let $\tau$ be a piecewise monotonic function from $J \rightarrow J$ with the fixed partition $\mathscr{G}_{N}$ consisting of the $N$ intervals $\left\{I_{1}, \ldots, I_{N}\right\}$. We define an induced motion on these intervals, referred to as the state space, as follows:

$$
\left\{x \in J: Z_{n}(x)=i\right\} \Leftrightarrow\left\{x \in J: \tau^{n}(x) \in I_{i}\right\},
$$

$n=0,1,2, \ldots$, where $Z_{n}: J \rightarrow\{1,2, \ldots, N\}$. If $\tau^{n}(x) \in Q$, the partition points of $g_{N}$, we make the convention that $Z_{n}(x)=i$, where $I_{i}$ is to the left of $x$. Then, for any $x$, the sequence (coding) of $Z_{n}$ 's is well defined. Let

$$
\begin{aligned}
I_{i_{0} i_{1} i_{2}} \ldots & =\left\{x \in J: x \in I_{i_{0}}, \tau(x) \in I_{i_{1}}, \tau^{2}(x) \in I_{i_{2}}, \ldots\right\} \\
& =\left\{x \in J: Z_{0}(x)=i_{0}, Z_{1}(x)=i_{1}, Z_{2}(x)=i_{2}, \ldots\right\} .
\end{aligned}
$$

In [7], sufficient conditions are presented which guarantee that the motion on the state space can be described by a Markov chain. We state the following lemma which is a modified version of Theorem 2 of [7]. 
LEMMA 4. Let $\tau: J \rightarrow J$ be piecewise monotonic from $J \rightarrow J$, and let $Z_{n}$ be the motion induced on the state space $\left\{I_{i}\right\}_{i=1}^{N}$. Let the random variable $Z_{0}$ have a distribution on $\{1, \ldots, N\}$ given by $m\left(I_{i}\right)$. Then the flow of distributions $\left\{\mu_{n}\right\}_{n>0}$ induced by the random variables $\left\{Z_{n}\right\}_{n>0}$ can be described by $a$ Markov chain if the following two conditions hold:

(a) $\left.\tau\right|_{I_{i j}}=\alpha_{i j} x+\beta_{i j}, 1 \leqslant i, j \leqslant N$,

(b) $\tau\left(I_{i j}\right)=I_{j}$ a.e. if $I_{i j} \neq \varnothing, 1 \leqslant i, j \leqslant N$.

If (a) and (b) are fulfilled, the transition probabilities associated with the Markov chain are

$$
P\left\{Z_{n+1}=j / Z_{n}=i\right\}=\frac{m\left(I_{i j}\right)}{m\left(I_{i}\right)} .
$$

The requirement that $\tau$ is piecewise linear will guarantee (a). Further, if we assume that $\tau \in \mathcal{C}$ then (b) will be satisfied. As well

$$
\tau\left(I_{i}\right)=\bigcup_{q=1}^{n_{i}} I_{i_{q}}, \quad n_{i} \text { is finite. }
$$

For any piecewise continuous $\tau \in \mathcal{C}$, let $\tau_{L}$ be the piecewise linear approximation to $\tau$ obtained by joining the endpoints of $\tau$ by straight lines. Clearly $\left|\tau_{L}^{\prime}\right|>0$. Since $\tau$ takes partition points into partition points, so does $\tau_{L}$ and (5) remains valid for $\tau_{L}$. Since $\tau_{L}$ satisfies (a) and (b) we know that it induces a Markov chain on the state space.

Let $\mathcal{P}$ denote the transition matrix $\left(p_{i j}\right)$ of the Markov chain induced by $\tau_{L}$, i.e. $p_{i j}=m\left(I_{i j}\right) / m\left(I_{j}\right)$. By Lemma 4 we know that the matrix $\mathscr{P}^{(n)}$, $\mathscr{P} \cdot \mathscr{P} \ldots \mathscr{P}, n$ times, represents the $n$-state transition matrix, i.e. $\mathscr{P}_{i j}^{(n)}=$ $P\left\{Z_{n}=j / Z_{0}=i\right\}$. Thus,

$$
\mathscr{P}(n)=\frac{P\left\{Z_{0}=i, Z_{n}=j\right\}}{P\left\{Z_{0}=i\right\}}=\frac{m\left\{x \in J: x \in I_{i}, \tau^{n}(x) \in I_{j}\right\}}{m\left(I_{i}\right)},
$$

and we obtain

$$
\mathscr{P}_{i j}^{(n)}=\frac{m\left\{I_{i} \cap \tau^{-n}\left(I_{j}\right)\right\}}{m\left(I_{i}\right)} .
$$

We are now ready to prove the following result, the purpose of which is to facilitate the checking of the communication condition.

Proposition 2. Let $\tau \in \mathcal{C}$. Let $\tau_{L}$ be its piecewise linear approximation and let $\mathcal{P}$ be the transition matrix of the Markov chain induced by $\tau_{L}$. If there exists an integer $n$ such that $\mathcal{P}^{(n)}>0$ (all entries of $\mathcal{P}^{(n)}$ are $>0$ ), then $\tau_{L}$ has the communication property.

Proof. Let $\mathscr{P}^{(n)}>0$. Then, by (6), $m\left(I_{i} \cap \tau_{L}^{-n}\left(I_{j}\right)\right)>0$. But

$$
\tau_{L}^{n}\left(I_{i} \cap \tau_{L}^{-n}\left(I_{j}\right)\right)=\tau_{L}^{n}\left(I_{i}\right) \cap I_{j}
$$


It therefore follows that

$$
m\left(\tau_{L}^{n}\left(I_{i}\right) \cap I_{j}\right)>0
$$

Since $\left|\tau_{L}^{\prime}\right|>0,\left|\left(\tau_{L}^{n}\right)^{\prime}\right|>0$. Also since $\tau_{L}$ takes partition points into partition points, so does $\tau_{L}^{n}$. Hence $\tau_{L}^{n}\left(I_{i}\right)=\bigcup_{q=1}^{l_{i}} I_{i_{q}}$, and (7) implies that $\tau_{L}^{n}\left(I_{i}\right) \supset I_{j}$. Recalling that $\tau$ has the same endpoints as $\tau_{L}$, we obtain $\tau^{n}\left(I_{i}\right)=\tau_{L}^{n}\left(I_{i}\right) \supset I_{j}$. Q.E.D.

EXAMPLE 3. For Example 1,

$$
\mathscr{P}=\left(\begin{array}{llll}
0 & 0 & .5 & .5 \\
0 & 0 & 1 & 0 \\
0 & .5 & .5 & 0 \\
1 & 0 & 0 & 0
\end{array}\right) .
$$

$\mathscr{P}^{(6)}>0$, and we have the communication property, which we already knew by checking directly.

COROLlary 1. Let $\tau \in \mathcal{C}$. If $\mathcal{P}(n)>0$, then $\tau_{L}$ has a unique absolutely continuous invariant measure.

Proof. Theorem 1.

We now prove a matrix theoretic result which will be needed for the central limit theorem of $\$ 5$, and which is useful in its own right.

Proposition 3. Let $\tau \in \mathcal{C}$ have a fixed point and let $\tau_{L}$ be the piecewise linear approximation to $\tau$ with respect to the basic partition $G^{L}$. Let $G_{M}$ be a finer partition of $J$ then $I$, and let $\bar{\tau}_{L}$ be the piecewise linear approximation to $\tau$ with respect to $G_{M}$. Assume that $\tau$ takes partition points of $G_{M}$ into partition points of $g_{M}$. Let ${ }_{M} \mathcal{P}$ be the transition matrix of the Markov chain induced by $\bar{\tau}_{L}$ for the partition $\mathscr{G}_{M}$, i.e. ${ }_{M} \mathscr{P}_{i j}=\left({ }_{M} p_{i j}\right)$, where

$$
{ }_{M} p_{i j}=\frac{m\left(I_{i}^{M} \cap \bar{\tau}_{L}^{-1}\left(I_{j}^{M}\right)\right)}{m\left(I_{i}^{M}\right)}, \quad I_{i}^{M}, I_{j}^{M} \in \mathscr{G}_{M} .
$$

If there exists an integer $n$ such that $\mathscr{P}^{(n)}>0$, then the larger matrix ${ }_{M} \mathcal{P}$ also has the property that there exists $n^{\prime}=n^{\prime}(M)$ such that ${ }_{M}^{\mathscr{P}^{\left(n^{\prime}\right)}}>0$.

Proof. Let $\tau \in \mathcal{C}$ and assume $\exists n \ni \mathscr{P}^{(n)}>0$. From Proposition 2 and Lemma 3, it follows that there exists a dense orbit in $J$. We shall now improve this result in the following way: for each $I_{i}^{M} \in \mathscr{G}_{M}, y_{j} \in I_{j}^{M} \in \mathscr{G}_{M}$, and any $\varepsilon_{j}>0, i, j=1,2, \ldots, M, \exists x_{i j} \in I_{i}^{M}$ and an $n$, independent of $i$ and $j$, such that

$$
\left|\tau^{n}\left(x_{i j}\right)-y_{j}\right|<\varepsilon_{j}
$$

for all $i, j=1, \ldots, M$. 
To show this, we proceed as in Lemma 3, where the coding is now with respect to the partition $g_{M}$. Let $S_{j}$ be the symbol corresponding to the intervals containing $y_{j}, \tau\left(y_{j}\right), \ldots, \tau^{m_{1}}\left(y_{j}\right)$, where $m_{1}$ will be chosen later.

Since $\tau$ has a fixed point, $\tau$ takes partition points into partition points, and $\left|\tau^{\prime}\right|>0$ (recall $\left|\left(\tau^{l}\right)^{\prime}\right|>1$ ), it follows that there is a symbol $\alpha$ with the property that $\tau(I(\alpha)) \supset I(\alpha)$, where $I(\alpha) \in \mathscr{G}_{M}$. Let $\alpha_{i}$ correspond to $I_{i}^{M} \in$ $g_{M}$, and form the sequences

$$
\left\langle x_{i j}\right\rangle=. \alpha_{i} \cdots \alpha \cdot \alpha \cdots S_{j} \cdots,
$$

where $S_{j}$ starts in the $n$th place, $i, j=1,2, \ldots, M$. Since there exists a dense orbit, we can go from $\alpha_{i}$ to $\alpha$ and from $\alpha$ to $\alpha_{j}$ in a finite number of iterations. To choose the same $n$ for each $x_{i j}$, a sufficient number of $\alpha$ 's are inserted in each sequence so that each $S_{j}$ starts at the $n$th place, independent of $i, j$.

Now, under the assumption inf $\left|d \tau^{l} / d x\right| \geqslant d>1$, we have

$$
\begin{aligned}
\left|\tau^{n}\left(x_{i j}\right)-y_{j}\right| & \leqslant \frac{1}{d}\left|\tau^{n+l}\left(x_{i j}\right)-\tau^{l}\left(y_{j}\right)\right| \\
& \leqslant \frac{1}{d^{m}}\left|\tau^{n+m l}\left(x_{i j}\right)-\tau^{m l}\left(y_{j}\right)\right| \\
& \leqslant \frac{2 C}{d^{m}}<\varepsilon_{j},
\end{aligned}
$$

if $m_{1}=m l$ is sufficiently large, where $C=\max _{x \in J} \tau(x)$. From this, it follows that for all $i, j=1, \ldots, M, \exists n$ such that $\tau^{n}\left(I_{i}^{M}\right) \cap$ int $I_{j}^{M} \neq \varnothing$. Piecewise continuity of $\tau$ then establishes: $m\left(\tau^{n}\left(I_{i}^{M}\right) \cap I_{j}^{M}\right)>0$. Since $\tau$ takes partition points of $G_{M}$ into partition points, it follows that $\tau^{n}\left(I_{i}^{M}\right) \supset I_{j}^{M}, j=$ $1, \ldots, M$, and thus $\tau^{n}\left(I_{i}^{M}\right) \supset \cup_{j} I_{j}^{M}=J$. Noticing that $\bar{\tau}_{L}\left(I_{i}^{M}\right)=\tau\left(I_{i}^{M}\right)$, we have $\bar{\tau}_{L}^{n}\left(I_{i}^{M}\right) \supset J, i=1, \ldots, M$. It also follows that

$$
{ }_{M} p_{i j}^{(n)}=\frac{m\left(I_{i}^{M} \cap \bar{\tau}_{L}^{-n}\left(I_{j}^{M}\right)\right)}{m\left(I_{i}^{M}\right)}>0
$$

and so ${ }_{M}^{\mathcal{P}^{(n)}}>0$. Q.E.D.

COROLlaRY 2. Let $\tau$ have the property that the set of eventually periodic points is dense in $J$. Then for any nonempty open set $U \subset J$, there exists an integer $n \geqslant 1$ such that $\tau^{n}(U)=J$. (The condition $\tau^{n}(U)=J$ implies 'strong transitivity' [11].)

Proof. Since the eventually periodic points are dense in $J$, the partition $\mathscr{G}_{M}$ can be chosen sufficiently fine so that $I_{i}^{M} \subset U$ for some $i$. By the theorem, $\exists n \exists \tau^{n}\left(I_{i}^{M}\right)=J$. Q.E.D.

EXAMPLE 4. Consider $\tau$ defined in Example 1 with $q_{M}$, a partition of $[1,5]$ having $M=4.2^{n}$ equal subintervals, where $n \geqslant 1$. Here, obviously $\tau=\tau_{L}$. It can readily be checked that $\tau$ takes the partition points of $G_{M}$ into the 
partition points of $g_{M}$. In Example 3, it was seen that for the basic partition $g=\{(1,2),(2,3),(3,4),(4,5)\}, \mathcal{P P}^{(6)}>0$. Thus, Proposition 2 implies that the $M \times M$ matrix ${ }_{M} \mathscr{P}$ also has the property that ${ }_{M} \mathscr{P}^{(j)}>0$ for some $j$. The matrix $_{M} \mathcal{P}$ consists of sixteen equal sized $2^{n} \times 2^{n}$ blocks and is of the form

$$
\left(\begin{array}{llll}
0 & 0 & B_{13} & B_{14} \\
0 & 0 & 0 & I^{\prime} \\
0 & B_{32} & B_{33} & 0 \\
I^{\prime} & 0 & 0 & 0
\end{array}\right),
$$

where

$$
B_{13}=\left(\begin{array}{c}
H \\
\hdashline 0
\end{array}\right), \quad B_{14}=\left(\begin{array}{c}
0 \\
\hdashline H
\end{array}\right),
$$

and

$$
H=\left(\begin{array}{llll}
.5 .5 & & & \\
& .5 .5 & & \\
& & \ddots & .5 .5
\end{array}\right)
$$

is an $2^{n-1} \times 2^{n}$ matrix; and

$$
B_{32}=\left(\begin{array}{c}
G \\
\hdashline 0
\end{array}\right), \quad B_{33}=\left(\begin{array}{c}
0 \\
\hdashline G
\end{array}\right),
$$

where the $2^{n-1} \times 2^{n}$ matrix $G$ is

$$
G=\left(\begin{array}{lll} 
& .5 .5 & .5 .5 \\
.5 .5 &
\end{array}\right]
$$

and the $2^{n} \times 2^{n}$ matrix

$$
I^{\prime}=\left(\begin{array}{lllll} 
& & & & \\
& & & & \\
& & & &
\end{array}\right) .
$$

If $n=4$, say, ${ }_{M}^{\mathcal{P}}$ has 4096 entries of which only 192 are nonzero. Nonetheless, Proposition 2 guarantees that eventually all entries will be greater than 0 .

In matrix theoretic language, Proposition 3 presents a condition which ensures that the special matrices ${ }_{M} \mathcal{P}$ are primitive, i.e. there exists an $n$ such that all entries of ${ }_{M}{ }^{\mathcal{P}^{(n)}}$ are positive. Proposition 3 can be employed in the 
following way as well: given a $K \times K$ matrix $Q$; if from $Q$ we can reconstruct a piecewise linear function $\tau$, whose associated transition probability matrix is $Q$, and if $\tau$ has a fixed point, takes partition points into partition points and has slopes as described earlier, then $Q$ is primitive. We will not pursue these ideas further here.

We observe that it is always possible to find finer and finer partitions of $J$ for which $\tau$ takes partition points into partition points if the eventually periodic points of $\tau$ are dense in $J$. In [4], it is shown that this is true for the class of piecewise linear functions which have integer slopes $\left\{d_{i}, i=\right.$ $1, \ldots, N\}$ and rational intercepts.

It is also easy to see that if $\tau$ has its eventually periodic points dense in $J$, then so does any topologically conjugate transformation of the form $h \circ \tau \circ h^{-1}$, where $h: J \rightarrow J$ is a homeomorphism.

We now present two examples of nonlinear functions which have the property that their eventually periodic points are dense in the domain.

EXAMPLE 5 . Let $\tau:[0,1] \rightarrow[0,1]$ be defined by

$$
\tau(x)= \begin{cases}2 x+\frac{1}{2}, & x \in\left[0, \frac{1}{4}\right], \\ -x+\frac{5}{4}, & x \in\left[\frac{1}{4}, \frac{1}{2}\right], \\ -2 x+\frac{7}{4}, & x \in\left[\frac{1}{2}, \frac{3}{4}\right] \\ -x+1, & x \in\left[\frac{3}{4}, 1\right] .\end{cases}
$$

This is a compressed version of $\tau$ in Example 1; its unique invariant density $f$ is given by $\left\{\frac{2}{7}, \frac{1}{7}, \frac{2}{7}, \frac{2}{7}\right\}$ on the intervals $\left(0, \frac{1}{4}\right),\left(\frac{1}{4}, \frac{1}{2}\right),\left(\frac{1}{2}, \frac{3}{4}\right),\left(\frac{3}{4}, 1\right)$, respectively. Let $h:[0,1] \rightarrow[0,1]$ be the homeomorphism defined by $h(x)=\sqrt{x}$. Then

$$
\tau_{1}(x)=h^{-1} \circ \tau \circ h(x)= \begin{cases}\left(2 \sqrt{x}+\frac{1}{2}\right)^{2}, & x \in\left[0, \frac{1}{16}\right], \\ \left(-\sqrt{x}+\frac{5}{4}\right)^{2}, & x \in\left[\frac{1}{16}, \frac{1}{4}\right], \\ \left(-2 \sqrt{x}+\frac{7}{4}\right)^{2}, & x \in\left[\frac{1}{4}, \frac{9}{16}\right], \\ (-\sqrt{x}+1)^{2}, & x \in\left[\frac{9}{16}, 1\right],\end{cases}
$$

is topologically conjugate to $\tau$ and, therefore, has eventually periodic points which are dense in $[0,1]$ since $\tau$ does. The unique invariant density for $\tau_{1}$ is $f_{1}(x)=f(\sqrt{x}) / 2 \sqrt{x}$. The functions $\tau$ and $\tau_{1}$ are sketched in Figure 2(a), (b). 
EXAMPLE 6. For $\tau$ and $h$ as in Example 3, define $\tau_{2}=h \circ \tau \circ h^{-1}$. Then

$$
\tau_{2}(x)= \begin{cases}\left(2 x^{2}+\frac{1}{2}\right)^{1 / 2}, & x \in\left[0, \frac{1}{2}\right], \\ \left(-x^{2}+\frac{5}{4}\right)^{1 / 2}, & x \in\left[\frac{1}{2}, \frac{\sqrt{2}}{2}\right] \\ \left(-2 x^{2}+\frac{7}{4}\right)^{1 / 2}, & x \in\left[\frac{\sqrt{2}}{2}, \frac{\sqrt{3}}{2}\right], \\ \left(-x^{2}+1\right)^{1 / 2}, & x \in\left[\mid \frac{\sqrt{3}}{2}, 1\right] .\end{cases}
$$

The unique invariant density under $\tau_{2}$ is $f_{2}(x)=f\left(x^{2}\right) 2 x$. The function $\tau_{2}(x)$ is sketched in Figure 2(c).

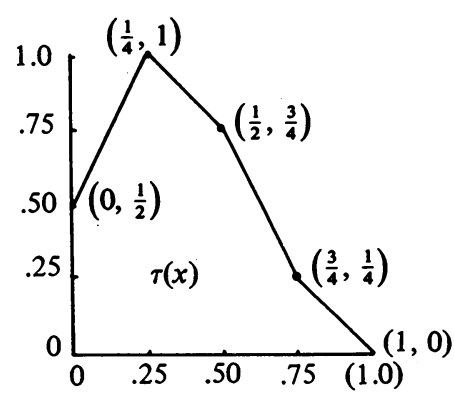

(a)

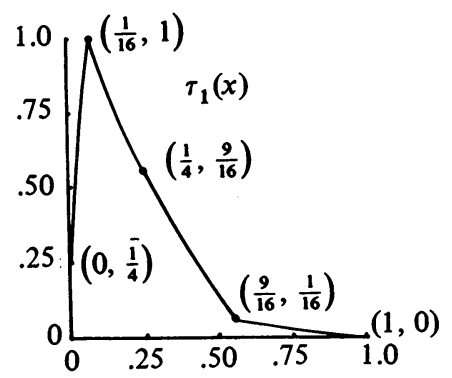

(b)

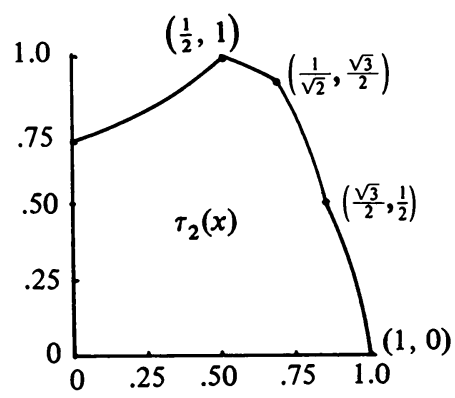

(c)

FIgURE 2

We note that both $\tau_{1}$ and $\tau_{2}$ have regions with slopes less than 1 in absolute value $\left(\tau_{1}^{\prime}(1)=0, \tau_{2}^{\prime}(0)=0\right)$, and yet have absolutely continuous invariant measures (in fact, unique ones). This is interesting in view of the counterexample in [1], where a transformation which has slope greater than 1 except 
at the point $(0,0)$, where the slope is 1 , has no absolutely continuous invariant measure. It is easy to see, however, that the counterexample has the property that any function topologically conjugate to it will also have slope $<1$ at $(0,0)$.

Furthermore, we now show that $\tau_{2}$ has the property that every iterate has a region with slope $<1$ in absolute value, and thus the existence of an absolutely invariant measure under $\tau_{2}$ cannot be proved using Theorem 1 of [1]. Since

$$
\left.\tau^{n}(x)\right|_{\left(a_{i-1}, a_{i}\right)}=c_{i} x+d_{i}, \quad i=1,2, \ldots, N,
$$

where $c_{i}$ is an integer and $d_{i}$ a rational number, both depending on $n$, it follows that

$$
\tau_{2}^{n}(x)=\sqrt{c_{i} x^{2}+d_{i}}, \quad x^{2} \in\left(a_{i-1}, a_{i}\right)
$$

and

$$
\left(\tau_{2}^{n}\right)^{\prime}(x)=\frac{c_{i} x}{\sqrt{c_{i} x^{2}+d_{i}}}, \quad x^{2} \in\left(a_{i-1}, a_{i}\right)
$$

which can be made arbitrarily small in absolute value for $x$ near 0 if $d_{1} \neq 0$. Now $d_{i}=0$ only if $n$ is a multiple of 4 since the orbit of 0 under $\tau$ has period 4. Thus, $\tau_{2}^{n}$ has an interval with arbitrarily small slope if $n$ is not a multiple of 4. But,

$$
\frac{d \tau_{2}^{4 m}}{d x}=\frac{d}{d x}\left[\tau_{2}\left(\tau_{2}^{4 m-1}(x)\right)\right]=\frac{d \tau_{2}(y)}{d y} \frac{d \tau_{2}^{4 m-1}(x)}{d x} .
$$

The function $d \tau_{2} / d y$ is bounded, and by the foregoing argument $\left(d \tau_{2}^{4 m-1} / d x\right)(x)$ can be made arbitrarily small near 0 . Hence, the same is true for $\tau_{2}^{4 m}$, and we conclude that every iterate of $\tau_{2}$ has a region on which its slope $<1$ in absolute value. It would be of interest to know if there exists a piecewise linear function which has both this property and an absolutely continuous invariant measure.

5. A central limit theorem. Let $\tau \in \mathcal{C}$ have a fixed point and let its eventually periodic points be dense in $J$. Then there exists an infinite sequence of finer and finer partitions $\left\{g_{k}: k \geqslant K\right\}$, such that for each $k>K$, $\tau$ takes partition points of $G_{k}$ into partition points of $g_{k}$, and $\tau$ satisfies the communication property with respect to $\mathscr{G}_{k}$. Let $\tau_{k}$ be the piecewise linear approximation to $\tau$ with respect to $G_{k}$. Then $\tau_{k}$ induces a Markov chain with transition matrix ${ }_{k} \mathscr{P}$ which is primitive. This ensures the existence of an invariant measure $\pi_{k}$ on the $k$ intervals of $g_{k}$, where $\pi_{k}$ is the unique solution of $\left(\pi_{k}\right)_{k} \mathscr{P}=\pi_{k}$.

Since $\tau \in \mathcal{C}$, we know that it has a unique absolutely continuous invariant 
measure $\pi$ on the infinite state space $J$. We can, therefore, invoke Theorem 1 of [6], which ensures that $\pi_{k} \rightarrow \pi$ in $\mathfrak{L}_{1}$. Let $A$ be any measurable set in $J$. Then

$$
\pi(A)=\lim _{k \rightarrow \infty} \pi_{k}(A)=\lim _{k \rightarrow \infty} \sum_{j(A)} \pi_{k}\left(I_{j}^{k}\right),
$$

where $j(A)=\left\{j: I_{j}^{k} \subset A, I_{j}^{k} \in \mathscr{G}_{k}\right\}$.

Let us now define the family of random variables $\left\{X_{0}, X_{1}, X_{2}, \ldots\right\}$, where $X_{i}=\tau^{i}\left(X_{0}\right)$ and $X_{0}$ has the distribution obtained from the invariant measure $\pi$. This is clearly a stationary sequence of random variables. Our aim is to show that this sequence is uniformly mixing. To that end, let us define a transition operator on the infinite space $J$ in the following way:

$$
P(x, A)=\lim _{k \rightarrow \infty} \sum_{j \in j(A)}{ }_{k} P_{i_{k} j}
$$

and $i_{k}=i_{k}(x)$ denotes the set $I_{i_{k}}^{k} \in g_{k}$ which contains the point $x$.

A sufficient condition for $\left\{X_{0}, X_{1}, \ldots\right\}$ to be uniformly mixing is given by equation (19.1.7) of [8]:

$$
\sup _{x, A}\left|P^{(n)}(x, A)-\pi(A)\right|<C \rho^{n},
$$

where $C$ and $\rho$ are constants, $0<\rho<1$, and the $n$-step transition probabilities $P^{(n)}(x, A)$ are given by

$$
P^{(1)}(x, A)=P(x, A), \quad P^{(n)}(x, A)=\int_{J} P^{(n-1)}(y, A) P(x, d y) .
$$

To prove (11), we proceed as follows:

$$
\begin{aligned}
\left|P^{(n)}(x, A)-\pi(A)\right|< & \left|P^{(n)}(x, A)-\sum_{j \in j(A)}{ }_{k} P_{i_{k} j}^{(n)}\right| \\
& +\left|\sum_{j \in j(A)}{ }_{k} P_{i_{k} j}^{(n)}-\pi_{k}(A)\right|+\left|\pi_{k}(A)-\pi(A)\right|,
\end{aligned}
$$

where $k$ denotes the partition $G_{k}$. In view of (9), the third term on the right-hand side of (13) vanishes as $k \rightarrow \infty$. The definition of $P^{(n)}(x, A),(10)$ and (13), ensures that the first term on the right-hand side goes to 0 as $k \rightarrow \infty$. Now, for the partition $G_{k}$, the transition ${ }_{k} \mathscr{P}$ matrix satisfies ${ }_{k} \mathscr{P}^{(l)}>$ 0 for some $l$. Hence, it follows [8, equation (19.1.2)] that there exist constants $C_{k}, \rho_{k}, 0<\rho_{k}<1$, such that for any $i_{k}$,

$$
\left|{ }_{k} P_{i k j}^{(n)}-\pi_{k}\left(I_{j}^{k}\right)\right|<C_{k} \rho_{k}^{n}
$$


From this, we get

$$
\begin{aligned}
\left|\sum_{j \in j(A)}{ }_{k} P_{i_{k} j}^{(n)}-\sum_{j \in j(A)} \pi_{k}\left(I_{j}^{k}\right)\right| & \leqslant \sum_{j \in j(A)}\left|{ }_{k} P_{i_{k} j}^{(n)}-\pi_{k}\left(I_{j}^{k}\right)\right| \\
& \leqslant \sum_{j=1}^{k} C_{k} \rho_{k}^{n}=k C_{k} \rho_{k}^{n},
\end{aligned}
$$

which goes to 0 as $n \rightarrow \infty$. Hence, (11) is established. Q.E.D.

For the class of functions $\tau$ under consideration, it follows from Corollary 2 that $\lim _{n \rightarrow \infty} \pi\left(\tau^{n} U\right)=1$ for all nonempty intervals $U \subset J$, with $\pi(U)>0$. Hence, by Theorem 2 of [12], $\pi$ is weak-mixing, and by Theorem 1 of [12], $\tau$ is Bernoulli under $\pi$. With this last result, it is shown in [9] that a central limit theorem is true for a class of real Hölder functions. The arguments are long and tedious. For our class of functions $\tau$, the uniform mixing condition (11) was obtained very easily, and it allows us to invoke Theorem 19.1.2 of [8] directly, to obtain a central limit theorem which is true for all real-valued measurable functions on $J$. We state the result.

THEOREM 2. Let $\tau \in \mathcal{C}$ have a fixed point and let its eventually periodic points be dense in $J$. Let $f(x)$ be a real-valued measurable function on J, and let $g(x)$ be the density of the unique invariant measure $\pi$. If

$$
E\left|f\left(X_{0}\right)\right|^{2}=\int_{J} f^{2}(x) g(x) d x<\infty
$$

and

$$
\begin{aligned}
\sigma^{2}= & E\left\{f\left(X_{0}\right)-E f\left(X_{0}\right)\right\}^{2} \\
& +2 \sum_{j=0}^{\infty} E\left\{\left(f\left(X_{0}\right)-E f\left(X_{0}\right)\right)\left(f\left(X_{j+1}\right)-E f\left(X_{j+1}\right)\right)\right\} \neq 0,
\end{aligned}
$$

where $E$ denotes the expectation operator with respect to $g(x) d x$, then for any initial distribution $v$ on $J$,

$$
\lim _{n \rightarrow \infty} P_{\nu}\left\{\frac{1}{\sigma \sqrt{n}} \sum_{j=0}^{n-1}\left[f\left(X_{j}\right)-E f\left(X_{j}\right)\right]<z\right\}=\frac{1}{\sqrt{2 \pi}} \int_{-\infty}^{z} e^{-u^{2} / 2} d u,
$$

where $P_{\nu}$ denotes the probability measure induced by $\nu$.

REMARK. There is a large class of chaotic functions [2] which satisfy the conditions of Theorem 2 [4]. It has recently become clear in the biological literature [3], [13] pertaining to chaotic functions, that chaotic orbits (basically orbits which do not even asymptotically approach any periodic cycle) must be treated probabilistically. Theorem 2 corroborates that approach. Indeed, if we 
let $f(x)=x$ and define

$$
S_{n}=\frac{1}{\sigma \sqrt{n}} \sum_{i=0}^{n-1}\left(\tau^{i}\left(X_{0}\right)-m(J)\right),
$$

then the sequence of random variables $S_{0}, S_{1}, S_{2}, \ldots$ approaches normalized Brownian motion in distribution [15].

6. Piecewise constant invariant measures. For $\tau \in \mathcal{C}$, it is usually difficult to find its unique absolutely continuous invariant density $f$. One can, however, approximate $f$ in the $\mathcal{E}_{1}$ sense as closely as desired with the aid of [6, Theorem 1]. In the special case that $\tau \in \mathcal{C}$ is piecewise linear and has a fixed point, we can find $f$ directly; it is piecewise constant, and the solution of a matrix equation.

Theorem 3. Let $\tau \in \mathcal{C}$ be piecewise linear and let it have a fixed point. Then the unique absolutely continuous invariant density under $\tau$ is piecewise constant.

Proof. We claim that the equation

$$
P_{\tau} f=f
$$

has a solution of the form $f(x)=\sum_{l=1}^{N} e_{l} \chi_{I_{l}}(x)$, where the partition is $\mathscr{G}=$ $\left\{I_{i}\right\}_{i=1}^{N},\left\{e_{i}\right\}_{i=1}^{N}$ is a set of positive constants, and $\chi_{A}(x)$ is the characteristic function of $A$. With the requirement that $\sum_{l=1}^{N} e_{l}=1, f(x)$ becomes the unique invariant density under $\tau$.

If such an $f(x)$ satisfies (16), we have, for $x \in I_{j}$,

$$
e_{j}=P_{\tau}\left(\sum_{l=1}^{N} e_{l} \chi_{I_{l}}(x)\right)=\sum_{l=1}^{N} e_{l} P_{\tau}\left(\chi_{I_{l}}(x)\right) \text {. }
$$

From the definition of $P_{\tau}$, we obtain [1]

$$
e_{j}=\sum_{l=1} e_{l} \sum_{i=1}^{N} \chi_{I_{l}}\left(\tau_{i}^{-1}(x)\right)\left|\frac{d \tau_{i}^{-1}(x)}{d x}\right| \chi_{\tau_{i}\left(I_{i}\right)},
$$

where $\tau_{i}$ is the restriction of $\tau$ to the interval $I_{i}$. Let, for $x \in I_{j}$

$$
a_{j l}=\sum_{i=1}^{N} \chi_{I_{l}}\left(\tau_{i}^{-1}(x)\right)\left|\frac{d \tau_{i}^{-1}(x)}{d x}\right| \chi_{r_{i}\left(I_{i}\right)}
$$

Then

$$
e_{j}=\sum_{l=1}^{N} e_{l} a_{j l}
$$

where the equality in $x$ is true except at possibly a finite number of points. We claim

$$
a_{j l}=\left|\frac{d \tau_{l}^{-1}}{d x}\right| \delta_{j l}
$$


where $\delta_{j l}=1$ if $I_{j} \subset \tau_{l}\left(I_{l}\right)$ and 0 otherwise, i.e.,

$$
a_{j l}= \begin{cases}\left|\tau_{l}^{\prime}\right|^{-1}, & \text { if } I_{j} \subset \tau_{l}\left(I_{l}\right), \\ 0, & \text { otherwise. }\end{cases}
$$

To see this, note that the $i$ th term occurs in (17) with nonzero value only if $x \in I_{j}, x \in \tau_{i}\left(I_{i}\right)$ and $\tau_{i}^{-1}(x) \in I_{l}$, i.e., if

$$
I_{j} \subset \tau_{i}\left(I_{i}\right) \text { and } \tau_{i}^{-1}\left(I_{j}\right) \subset I_{l}
$$

or

$$
\tau_{i}^{-1}\left(I_{j}\right) \subset I_{i} \text { and } \tau_{i}^{-1}\left(I_{j}\right) \subset I_{l},
$$

which implies that $i=l$. Using this in (18) yields (20).

Let $E=\left(e_{1}, e_{2}, \ldots, e_{N}\right)^{t}$, where $t$ denotes transpose, and define the $N \times$ $N$ matrix $A=\left(a_{j l}\right)$. Thus equation (17) has a solution if

$$
E=A E \text {, }
$$

where $\sum_{l=1}^{N} e_{l}=1$, has a solution. Note that the system of linear equations (21) is equivalent to the system

$$
E^{t}=E^{t} A^{t}
$$

where $A^{t}=\left(a_{l j}\right), a_{l j}=\left|\tau_{j}^{\prime}\right|^{-1} \delta_{l j}$. Recall from $\S 4$ that $\left(a_{l j}\right)$ is the transition matrix for the Markov chain induced by $\tau$ on the state space 9 . Since $\tau \in \mathcal{C}$, $A^{t}$ is irreducible. The existence of a fixed point implies there exists an aperiodic state of the Markov chain. Hence, (22) has a solution, and therefore (21) has a solution. Q.E.D.

We note from the proof of the theorem that it is not necessary for $\tau$ to have a fixed point. What is required is that the matrix $A$ induced by $\tau$ be primitive.

REMARKS. (1) The class of transformations for which the unique absolutely continuous invariant measure can be computed exactly can be extended to all the transformations which are topologically conjugate to the piecewise linear transformations in $\mathcal{C}$ possessing fixed points.

(2) It follows from the proof of the theorem that on any segment $I_{i}$, $i=1,2, \ldots, N$, the function $\tau_{i}$ can be replaced by a linear function with the same domain and range, and slope equal to $-\tau_{i}^{\prime}$ without altering the invariant measure. Thus the theorem gives $2^{N}$ piecewise linear transformations all of which have the same invariant measure.

(3) If one attempts to verify the invariant measure obtained from the theorem by taking time averages, then it is important to choose a starting point which has a long orbit. The reader is referred to [4] for details on how the period of an orbit is related to a rational starting point. One should not necessarily conclude that a long decimal expansion yields a long orbit. For example in the piecewise linear function 


$$
\tau(x)= \begin{cases}5 x, & x \in[0,1), \\ -x+2, & x \in[1,2), \\ x+2, & x \in[2,3), \\ x-1, & x \in[3,4), \\ -5 x+25, & x \in[4,5],\end{cases}
$$

the starting point $x_{0}=\sqrt{8}$ to eight decimal places has a surprisingly small period. Following this orbit, one would never enter the segment $[1,2]$. However, the invariant measure for $\tau(x)$ is $(2,1,2,1,3)$ on the five segment partition.

ADDED IN PROOF. In the final paragraph of the proof of Theorem 3, we have tacitly assumed that the subintervals of the partition are all of equal length. Then $\left(a_{l j}\right)$ is a stochastic matrix. Since $\tau \in \mathcal{C},\left(a_{l j}\right)$ will also be primitive. Thus $\left(a_{l j}\right)$ has a unique fixed point.

We remark that $\left(a_{l j}\right)$ does not necessarily have to be stochastic to possess a unique fixed point. Consider, for example, the transformation

$$
\tau(x)=\left\{\begin{array}{l}
2 x, \quad 0<x \leqslant \frac{1}{2}, \\
-\frac{3}{2} x+\frac{7}{4}, \quad \frac{1}{2}<x<1 .
\end{array}\right.
$$

Let the partition consist of the two intervals $\left[\frac{1}{4}, \frac{1}{2}\right]$ and $\left[\frac{1}{2}, 1\right]$. Then the $2 \times 2$ matrix $\left(a_{l j}\right)$ is

$$
\left[\begin{array}{cc}
0 & \frac{2}{3} \\
\frac{1}{2} & \frac{2}{3}
\end{array}\right]
$$

possessing the unique fixed point $\left(1, \frac{3}{2}\right)$. The interval $\left[0, \frac{1}{4}\right]$ cannot be visited after a finite number of steps. Hence $\left(0,1, \frac{3}{2}\right)$ is the unique invariant density on the intervals $\left[0, \frac{1}{4}\right],\left[\frac{1}{4}, \frac{1}{2}\right]$ and $\left[\frac{1}{2}, 1\right]$, respectively.

\section{REFERENCES}

1. A. Lasota and J. A. Yorke, On the existence of invariant measures for piecewise monotonic transformations, Trans. Amer. Math. Soc. 186 (1973), 481-488.

2. T.-Y. Li and J. A. Yorke, Ergodic transformations from an interval into itself, Trans. Amer. Math. Soc. 235 (1978), 183-192.

3. J. Guckenheimer, G. Oster and A. Ipaktchi, The dynamics of density dependent population models, J. Math. Biol. 4 (1977), 101-147.

4. M. Scarowsky, A. Boyarsky and H. Proppe, Properties of piecewise linear chaotic functions, J. Nonlinear Analysis (to appear).

5. S. Wong, Some metric properties of piecewise monotonic mappings of the unit interval, Trans. Amer. Math. Soc. 246 (1978), 493-500.

6. T. Y. Li, Finite approximation for the Frobenius-Perron operator. A solution of Ulam's conjecture, J. Approximation Theory 17 (1976), 177-186. 
7. J. B. Moore and S. S. Sengupta, Existence and control of Markov chains in systems of deterministic motion, SIAM J. Control 13 (1975), 1103-1114.

8. I. A. Ibragimov and Yu. V. Linnik, Independent and stationary sequences of random variables, Wolters-Noordhoff, Groningen, 1971.

9. S. Wong, A central limit theorem for piecewise monotonic mappings of the unit interval, (preprint).

10. M. Marcus and H. Minc, A survey of matrix theory and matrix inequalities, Allyn and Bacon, Boston, Mass., 1964.

11. W. Parry, Symbolic dynamics and transformations of the unit interval, Trans. Amer. Math. Soc. 122 (1966), 368-378.

12. R. Bowen, Bernoulli maps of the interval, Israel J. Math. 28 (1977), 161-168.

13. R. May, Biological populations with nonoverlapping generations: stable points, stable cycles, and chaos, Science 186 (1974), 645-647.

14. C. Bowen, On axiom A diffeomorphisms, CBMS Regional Conf. Ser. Math., no. 35, Amer. Math. Soc., Providence, R. I., 1978.

15. P. Billingsley, Convergence of probability measures, Wiley, New York, 1968.

Department of Mathematics, Sir George Williams Campus, Concordia University, Montreal, Quebec, Canada 